\title{
Factor structure and internal reliability of cultural belief scales about colorectal cancer screening among Koreans in the Republic of Korea
}

\author{
Shin-Young Lee(i)
}

\begin{abstract}
Background: Culturally sensitive, reliable and valid cultural belief scales for colorectal cancer (CRC) screening in Koreans in the Republic of Korea are not available in the literature. The purpose of this study was to adapt and validate existing cultural belief scales for CRC screening in Koreans.

Methods: Individual interviews, expert reviews, and a pilot test were conducted for instrument adaptation, and a cross-sectional survey with 884 Koreans was conducted for instrument validation. Construct validity using exploratory and confirmatory factor analyses and reliability of the Korean version of the instruments were examined. Results: Exploratory factor analysis using c four factors that accounted for $48.12 \%$ of the variance. The validity and reliability of the cultural belief scales were supported by confirmatory factor analysis and Cronbach's alpha.

Conclusions: The results of the present study showed that the four-factor cultural belief scales were culturally sensitive, reliable and valid in Koreans. The final cultural belief scales could be used to identify cultural beliefs more accurately and specifically, as well as to develop effective interventions to increase CRC screening in Koreans in the Republic of Korea.
\end{abstract}

Keywords: Colorectal cancer, Culture, Koreans, Beliefs, Instruments

\section{Background}

Colorectal cancer (CRC) is the third most commonly diagnosed cancer among Koreans in the Republic of Korea [1]. CRC incidence rates increased significantly in Koreans aged 40 and older, and $90 \%$ of patients with CRC were aged 40 and older [2]. Compared with people in Western countries, Koreans aged younger than 50 years old were more likely to have CRC in the Republic of Korea; thus, the Korean National Cancer Committee recommended CRC screening for Koreans aged 45 to 80 [3]. CRC screening rates remain low despite the many opportunities available to Koreans for CRC screening tests by participating in the National Cancer Screening Program that the Korean National Health Insurance Service has provided or by visiting any medical facilities. The rates of CRC

Correspondence: shinyoung0114@gmail.com

Department of Nursing at Chosun University, 309 Pilmun-daero, Dong-gu, Gwangju 501-759, Republic of Korea

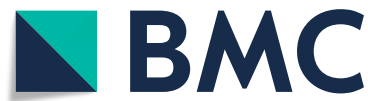

screening, including fecal occult blood test and colonoscopy, among Koreans aged 50 and older ranged from only 18.1 to $33.9 \%$ in the last 10 years $(2007-2016)$ in the Republic of Korea [4].

A review of the literature revealed that socio-demographic factors (e.g., low income) and beliefs (e.g., barriers) were risk factors Koreans who did not undergo CRC screening [5-7]. Particularly, Korean cultural beliefs, including (a) crisis health orientation toward health care use and (b) external influences on cancer screening behaviors (e.g., familism or fatalism), contributed in part to low CRC screening test utilizations among Korean populations [8-14]. Few studies have performed detailed investigates on how cultural beliefs influenced cancer screening behavior among Koreans in the Republic of Korea. Therefore, we extended the literature review by addressing cultural beliefs and cancer screening among Korean Americans in the US. First, the main barrier to

(c) The Author(s). 2018 Open Access This article is distributed under the terms of the Creative Commons Attribution 4.0 International License (http://creativecommons.org/licenses/by/4.0/), which permits unrestricted use, distribution, and reproduction in any medium, provided you give appropriate credit to the original author(s) and the source, provide a link to the Creative Commons license, and indicate if changes were made. The Creative Commons Public Domain Dedication waiver (http://creativecommons.org/publicdomain/zero/1.0/) applies to the data made available in this article, unless otherwise stated. 
cancer screening was the crisis health orientation toward health care use in Koreans (i.e., Koreans would seek medical care only if they had symptoms) $[10,11]$. Similarly, a lack of symptoms was the most common reason for not undergoing cancer screening among Korean Americans $[8,12]$. Second, external influences on cancer screening behaviors (e.g., family or fate) were revealed by studies in Korean populations [9, 12-14]. Although family members had encouraged cancer screening, many Koreans [9, 14] and Korean Americans [12, 13] had not sought cancer screening because of a lack of time due to family issues. Additionally, many Korean Americans believed that a life is predetermined since birth due to their traditional cultural beliefs stating that humans cannot control life and death; only God or an unknown supernatural power decides whether we are healthy or ill $[12,13]$. These beliefs cause some Korean Americans to ignore the value of cancer screening because they believe they will have cancer regardless of whether they undergo cancer screening [12].

The literature review identified that cultural beliefs importantly influenced cancer screening behaviors, and a Korean version of cultural belief scales for CRC screening was developed by Lee et al. [15] to be culturally sensitive for Korean Americans based on Russell's theoretical framework [16] combined with both the Cultural Assessment Model for Health [17] and the Powe Fatalism Model [18]. Given that other studies used only a few items to measure cultural beliefs regarding CRC screening behaviors among Korean Americans, Korean cultural belief scales for important cultural constructs, including cancer fatalism, health fatalism, health temporal orientation (crisis or preventive health orientation toward health care use) and personal control (internal or external control), were tested for construct validity and reliability and were found to have good performance for Korean Americans in the US [15]. However, currently, little research has been conducted to develop cultural belief scales for Koreans in the Republic of Korea, although cultural beliefs, such as crisis health orientation, familism, and fatalism, were revealed in cancer screening research with Koreans [9-11, 14]. Culturally sensitive, reliable and valid instruments measuring cultural beliefs are needed to obtain accurate study results, to identify behavioral risk factors and predictors of cancer screening behaviors, and to develop effective appropriate intervention programs to improve low CRC screening utilization among Koreans in the Republic of Korea. To develop reliable and valid cultural belief scales for Koreans, we used the cultural belief scales for Korean Americans [15] because both Korean populations have the same traditional Korean culture. However, constructs and items measuring cultural beliefs regarding CRC screening behaviors may be slightly different between Korean Americans in the US and Koreans in the Republic of Korea, and we needed instrument adaptation processes to make existing cultural belief scales culturally appropriate for Koreans regarding CRC screening behaviors. The purpose of this study was to adapt and validate the cultural belief scales regarding CRC screening behaviors among Koreans in the Republic of Korea.

\section{Methods}

This investigation was comprised of a descriptive qualitative study and a cross-sectional quantitative study through four steps (step 1: individual interviews, step 2: expert reviews, step 3: a pilot test, and step 4: a cross-sectional survey). Steps 1, 2, and 3 were used for instrument adaptation, and step 4 was conducted to test the psychometric properties of the instruments for Koreans in the Republic of Korea. This study's sample in several steps 1 to 4 consisted of Koreans who were aged 40 years and older.

\section{Instruments}

The initial instruments for cultural beliefs, including cancer fatalism, health fatalism, health temporal orientation, and personal control [15], were adapted, modified, and tested by the author of the present study to measure cultural beliefs about CRC screening while being culturally appropriate for Korean Americans in the US based on the cultural assessment for health [17] and cancer fatalism [18]. In Lee et al.'s study [15], Korean versions of culturally appropriate instruments were developed for Korean Americans, specifically for cancer fatalism (beliefs that death is inevitable when cancer is present), health fatalism (notions of fate, luck, destiny, and predetermination regarding diseases or health conditions), health temporal orientation (perspectives on current health beliefs and behaviors related to concerns about future health) and personal control (ability to plan activities to control or direct factors within the environment). Among the cultural belief constructs, the health temporal orientation scale consisted of items for crisis health orientation (symptoms are cues to seek health care) and preventive health orientation (identifying health problems early is important), and personal control consisted of items for internal control (the power to affect change lies within) and external control (fate, luck and change significantly impact outcomes). The initial Korean version of the cultural belief scales [15] consisted of a total of 52 items: cancer fatalism (15 items), health fatalism (15 items), health temporal orientation ( 5 items for preventive health orientation and 3 items for crisis health orientation), and personal control (4 items for internal control and 10 items for external control). The initial cultural belief scales established construct validity using exploratory factor analysis (EFA) and confirmatory factor analysis (CFA), and they had good reliability, 
with Cronbach's alpha coefficients of 0.77 to 0.88 [15]. All scales were measured on a five-point Likert scale from strongly disagree to strongly agree.

\section{Step 1: Individual interviews}

Individual interviews were conducted to investigate cultural belief concepts, including fatalism, health temporal orientation, and personal control regarding CRC screening, to ensure that the scales that were culturally sensitive and comprehensive for Koreans. The convenience sampling method was used to recruit participants from churches or community centers. A total of $35 \mathrm{Ko}$ reans (11 men and 24 women; 20 participants aged 40 to 64 and 15 participants aged 65 and older) participated in individual interviews.

During the interviews, participants discussed cultural beliefs related to CRC screening behavior via semi-structured, open-ended questions (Table 1). Each individual interview was audiorecorded and transcribed verbatim, and the data were coded into concepts. After reviewing the interview transcripts, the principal investigator compared concepts during individual interviews with the cultural belief scales to determine differences between them for instrument adaptation for Koreans.

Individual interviews revealed that many Koreans indicated fatalism related to cancer, health and disease, and life and death. The many participants mentioned that cancer was a deadly disease but that it could be treatable if it was found early. Most participants also believed in health fatalism, i.e., that people were born to be healthy or suffer from diseases based on their genes that were inherited from their parents. Regarding health temporal orientation, all of the participants agreed that it is important to detect health problems early, but most of them said that cancer could be prevented by their efforts, such as maintaining a healthy diet and regular exercise, although they did not consider going to medical facilities for cancer screening as a preventive behavior; CRC screening was considered part of the treatment process only when they had abnormal symptoms and disease. Regarding personal control, most of the participants

Table 1 Individual interview guide

\begin{tabular}{ll}
\hline Concepts & Discussion questions \\
\hline Cancer fatalism & $\begin{array}{l}\text { If people have colorectal cancer, will they die from } \\
\text { it regardless of what they do? What do you think } \\
\text { of fatalism and cancer? }\end{array}$ \\
Health fatalism & $\begin{array}{l}\text { How are external controls, such as fate, luck, } \\
\text { destiny, and predetermination, associated with } \\
\text { diseases or health conditions? }\end{array}$ \\
Health temporal & $\begin{array}{l}\text { How important do you think it is to detect health } \\
\text { orientation }\end{array}$ \\
$\begin{array}{ll}\text { problems early? What do you actively do to be } \\
\text { herceived control }\end{array}$ & $\begin{array}{l}\text { Who do you think has control over identifying } \\
\text { health problems early? }\end{array}$ \\
\hline
\end{tabular}

thought that cancer can develop from external forces that cannot be controlled but that people should make efforts to engage in healthy behaviors to prevent cancer. External influences from doctors, family, friends, and other influential individuals enabled them to obtain cancer screening.

All items in the cultural belief scales remained unchanged because Koreans in the Republic of Korea had almost the same cultural beliefs as Korean Americans. Based on two distinct fatalism versions that emerged from the individual interviews, two versions of the fatalism scales (cancer and health fatalism scales) were included in the present study, which were the same as those in the previous study [15]. However, in the present study, of the items in the initial health fatalism scale, three items ("I think it's fate to get cancer;" "I think cancer is always fatal;" and "I think there is little one can do to prevent cancer") were moved to the cancer fatalism scale because they were related to cancer based on findings from the individual interviews, and the results of the previous study [15] showed that these three health fatalism items were associated with cancer fatalism.

\section{Step 2: Expert reviews}

Six Korean researchers who were faculty members with doctoral degrees in health-related areas measured the content validity of the cultural belief scales. The experts independently rated content representativeness of each item on a four-point ordinal scale from not relevant to highly relevant. Item-level content validity was calculated by dividing the number of experts who assigned a rating of either 3 or 4 by the total number of experts, and scale-level content validity index/average was calculated by dividing the sum of the scores of item-level content validity by the number of items [19]. All item-level content validity and scale-level content validity values for the cultural belief scales were 1.0 because the experts rated all of the items either 3 or 4 . The experts suggested making unclear items clearer and understandable for Koreans, and items were modified accordingly.

\section{Step 3: A pilot test}

The purpose of the pilot test was to identify problems by testing the cultural belief scale with a large sample. Twenty-four participants (10 men and 14 women, 11 participants aged 40 and 64 and 13 participants aged 65 and older), who were recruited by convenience sampling methods from churches and community centers, were included in the pilot test. The participants completed the survey in 20 to $30 \mathrm{~min}$. The participants made suggestions, such as removing redundancies in the survey questionnaire, and the items were modified accordingly. 


\section{Step 4: A cross-sectional survey}

\section{Sample and data collection}

A total of 1025 Koreans were recruited from churches, community centers, stores, apartment communities, and companies in a metropolitan area in the Republic of Korea using convenience and chain referral sampling methods for the cross-sectional survey to assess psychometric properties of the final version of the cultural belief scale for CRC screening utilization among Koreans. Survey questionnaires were distributed in person to Koreans aged 40 and older. A total of 884 individuals participated in this study (response rate $=86.2 \%$ ). The absolute number of cases and the subject-to-variable ratio were used to determine the minimum sample size in factor analysis. A sample size of more than eight hundred cases in the present study was satisfactory for factor analysis based on a calculation of either the absolute number of cases or the subject-to-variable ratio [20-23].

The cultural belief scales in the questionnaire consist of four sections: cancer fatalism (18 items), health fatalism (12 items), health temporal orientation (3 items for crisis health orientation and 5 items for preventive health orientation), and personal control (4 items for internal control and 10 items for external control) (Additional file 1).

\section{Data analysis}

For factor analysis of the cultural belief scales, both EFA and CFA were performed using SPSS Version 24 [24] and AMOS 23 [25] in the present study. As complementary methods, EFA was conducted to identify a scale's underlying structure while CFA was performed to confirm the consistency between the scale and theoretical structure [26, 27]. In the EFA, the eigenvalues using the Kaiser criterion, scree plot, parallel analysis, and theoretical basis of cultural beliefs were considered to select the optimal number of factors. After EFA was conducted using principal component analysis with a varimax rotation, CFA was performed using structural equation modeling (SEM) with oblique (intercorrelated) factors. The data were also examined based on correlations among variables, path analysis, parameter estimates, and the model's fit to the observable data. Model fit was assessed by examining the relative chi-square (chi-square/degrees of freedom), root mean square error of approximation (RMSEA), goodness of fit index (GFI), standardized root mean square residual (SRMR), root mean square error of approximation (RMSEA), normed fit index (NFI), and comparative fit index (CFI). Factor loadings of each item were expected to be 0.40 or greater [28]. Reliability of all items in each scale was evaluated using Cronbach's coefficient alpha. Each of the subscales was expected to have reliability with a Cronbach's alpha of 0.70 or greater [29].

\section{Ethical considerations}

The institutional review board at the Chosun University approved the protocols in the present study. All participants were informed in verbal or written form about the purpose and procedures of the study, estimated risks and benefits, privacy protection and confidentiality, as well as their right to freely withdraw from the study at any time.

\section{Results \\ Sample characteristics}

Questionnaires completed by 884 individuals were analyzed in the present study (328 men and 556 women). The average participant age was 60 years $(M=59.87$; SD $=8.88$; range 40-87). A total of 282 (31.9\%) had a bachelors or higher degree, 767 (86.8\%) were married, and 453 (51.3\%) were employed.

\section{Construct validity}

EFA analysis demonstrated that the data were suitable because the Kaiser-Meyer-Olkin (KMO) result was 0.93 and the chi-square value for Bartlett's test of sphericity was 27,246.71 $(p<0.01)$. Using the EFA, four factors (factor 1: cancer fatalism; factor 2: health fatalism; factor 3: crisis health orientation and external control; and factor 4: preventive health orientation and internal control) were extracted and the latent root considered (eigenvalue $>1$ ) based on eigenvalues $>1$ using the Kaiser criterion, parallel analysis, scree plot, and theoretical basis (Table 2). Although the scree plot showed 4 clear changes in the slope, the eigenvalues $(>1)$ and parallel analysis using the Monte Carlo primary component analysis simulation resulted in more than 4 factors, but there is a single item under a factor as well as items with cross-factor loadings. The number of factors was reconsidered because (a) a single item is insufficient to measure a construct [30] and items loading on more than one factor are also suspect [31]; and (b) the number of factors that a parallel analysis produces may be an overestimate of the true dimensionality of the data [29]. Furthermore, it is important for the researcher to determine if the grouping of items in the EFA is theoretically consistent [31]. All evidence in the EFA was considered together for determining the number of factors underlying a set of item responses. The cumulative percentage of variance accounted for by these four factors was $48.12 \%$. Although some items were moved to different factors, item factor loadings of the EFA ranged from 0.26 to 0.81 . CFA using SEM was conducted with the four factors that were extracted from the EFA. Because the model did not fit the observed data, model respecification for all scales was needed. The results obtained from the CFA indicated that the model fit the data well, with adequate fit index values (Table 3 ). Although the fit 
Table 2 Rotated exploratory factor analysis of the cultural belief scales

\begin{tabular}{|c|c|c|c|}
\hline Factor 1: Cancer fatalism & Factor 2: Health fatalism & $\begin{array}{l}\text { Factor 3: Crisis health orientation } \\
\text { and external control }\end{array}$ & $\begin{array}{l}\text { Factor 4: Preventive health } \\
\text { orientation and internal control }\end{array}$ \\
\hline $\begin{array}{ll}\text { CF1 } & 0.53 \\
\text { CF2 } & 0.67 \\
\text { CF3 } & 0.61 \\
\text { CF4 } & 0.71 \\
\text { CF5 } & 0.72 \\
\text { CF6 } & 0.73 \\
\text { CF7 } & 0.69 \\
\text { CF8 } & 0.47 \\
\text { CF9 } & 0.74 \\
\text { CF10 } & 0.62 \\
\text { CF11 } & 0.76 \\
\text { CF12 } & 0.77 \\
\text { CF13 } & 0.79 \\
\text { CF14 } & 0.68 \\
\text { CF15 } & 0.69 \\
\text { CF16 } & 0.47 \\
\text { CF17 } & 0.31 \\
\text { CF18 } & 0.50 \\
\text { HF11 } & 0.46 \\
\text { HF12 } & 0.39\end{array}$ & $\begin{array}{ll}\text { HF1 } & 0.50 \\
\text { HF2 } & 0.59 \\
\text { HF3 } & 0.72 \\
\text { HF4 } & 0.75 \\
\text { HF5 } & 0.78 \\
\text { HF6 } & 0.75 \\
\text { HF7 } & 0.68 \\
\text { HF8 } & 0.69 \\
\text { HF9 } & 0.66 \\
\text { HF10 } & 0.58 \\
\text { EC9 } & 0.40 \\
\text { EC10 } & 0.44\end{array}$ & $\begin{array}{ll}\text { CHO1 } & 0.26 \\
\text { CHO2 } & 0.51 \\
\text { CHO3 } & 0.49 \\
\text { EC1 } & 0.50 \\
\text { EC2 } & 0.69 \\
\text { EC3 } & 0.60 \\
\text { EC4 } & 0.71 \\
\text { EC5 } & 0.67 \\
\text { EC6 } & 0.69 \\
\text { EC7 } & 0.63 \\
\text { EC8 } & 0.47\end{array}$ & $\begin{array}{ll}\text { PHO1 } & 0.80 \\
\text { PHO2 } & 0.81 \\
\text { PHO3 } & 0.79 \\
\text { PHO4 } & 0.66 \\
\text { PHO5 } & 0.46 \\
\text { IC1 } & 0.73 \\
\text { IC2 } & 0.71 \\
\text { IC3 } & 0.60 \\
\text { IC } 4 & 0.41\end{array}$ \\
\hline $\begin{array}{l}\text { Eigenvalue } \\
14.60\end{array}$ & 5.11 & 2.75 & 2.58 \\
\hline $\begin{array}{l}\text { Variance explained } \\
28.07\end{array}$ & 9.82 & 5.28 & 4.95 \\
\hline
\end{tabular}

CF cancer fatalism, $\mathrm{HF}$ health fatalism, EC external control, $\mathrm{CHO}$ crisis health orientation, $\mathrm{PHO}$ preventive health orientation, IC internal control

index values in the present study and the chi-square results of three factors (factors 1,2 , and 3 ) had large values $(P<0.05)$, indicating that the model does not fit the data with large residual effects, other fit indices should be considered because chi-square statistics are inflated by large sample sizes [32]. The data comply with the theoretical structure of cultural beliefs (cancer fatalism, health fatalism, crisis health orientation and external control, preventive health orientation, and internal control) for the GFI, SRMR, RMSA, NFI, and CFI results. Based on the CFA, the factor loadings of items ranged from 0.21 to 0.92 (Table 4). Three items in the EFA and 11 items in the CFA had a factor loading $<0.40$.

\section{Reliability}

The reliability of the cultural belief subscales was assessed by Cronbach's alpha coefficients, which are estimates of reliability (Table 5). All subscales demonstrated satisfactory reliability, with Cronbach's alpha values $>0.70$, according to the guidelines established by DeVellis [29].

\section{Discussion}

Although cultural beliefs influenced cancer screening behavior in Koreans [9-11, 14], culturally sensitive, reliable and valid instruments measuring cultural beliefs were not available for Koreans in the Republic of Korea. This is the first study that attempted to adapt and validate cultural belief scales for CRC screening in Koreans through several methodological steps. Individual interviews revealed that Koreans in the Republic of Korea had almost the same cultural beliefs regarding CRC screening as Korean Americans in the US [12]. Thus, the initial cultural belief scales were not changed significantly, except for the modification of words from unclear to clear and understandable and removal of redundancies from the sentences. The final cultural belief scales for CRC screening were culturally sensitive,

Table 3 Respecified measurement model fitness test results of the cultural belief subscales from confirmatory factor analysis

\begin{tabular}{|c|c|c|c|c|c|c|c|c|}
\hline Fitness index & $\times 2(p)$ & $\mathrm{df}$ & CMIN/DF & $\mathrm{GFI}$ & SRMR & RMSEA $(90 \% \mathrm{Cl})$ & $\mathrm{NFI}$ & $\mathrm{CFI}$ \\
\hline Criteria & $(>0.05)$ & & $<2$ & $\geq .95$ & $\leq .05$ & $\leq .05$ & $\geq .90$ & $\geq .95$ \\
\hline Cancer fatalism & $220.70(0.00)$ & 112 & 1.75 & 0.98 & 0.03 & $0.03(0.02-0.04)$ & 0.98 & 0.99 \\
\hline Health fatalism & $62.92(0.00)$ & 29 & 2.17 & 0.99 & 0.03 & $0.04(0.02-0.05)$ & 0.99 & 0.99 \\
\hline Crisis health orientation and external control & $46.64(0.01)$ & 25 & 1.87 & 0.99 & 0.01 & $0.03(0.02-0.05)$ & 0.99 & 0.99 \\
\hline Preventive health orientation and internal control & $20.25(0.38)$ & 19 & 1.07 & 0.99 & 0.01 & $0.01(0.00-0.03)$ & 0.99 & 1.00 \\
\hline
\end{tabular}

CMIN/DF chi-square minimum/degree of freedom, GFI goodness of fit index, SRMR standardized root mean square residual, RMSEA root mean square error of approximation, $\mathrm{Cl}$ confidence interval, $\mathrm{NFI}$ normed fit index, $\mathrm{CFI}$ comparative fit index 
Table 4 Confirmatory factor analysis of the cultural belief scales

\begin{tabular}{|c|c|c|}
\hline Scale and items & Corrected item-Total correlation & Factor loadings \\
\hline \multicolumn{3}{|l|}{ Factor 1 (cancer fatalism) } \\
\hline CF9. I think if someone is meant to have colorectal cancer, he/she will have colorectal cancer. & 0.71 & 0.80 \\
\hline $\begin{array}{l}\text { CF12. I think if someone has colorectal cancer and receives treatment for it, he/she will } \\
\text { probably still die from the colorectal cancer. }\end{array}$ & 0.74 & 0.80 \\
\hline $\begin{array}{l}\text { CF13. I think if someone was meant to have colorectal cancer, he/she will get colorectal } \\
\text { cancer regardless of what doctors and nurses tell him/her to do. }\end{array}$ & 0.76 & 0.80 \\
\hline $\begin{array}{l}\text { CF11. I think if someone gets colorectal cancer, he/she will die from it regardless of whether } \\
\text { it is detected early or late. }\end{array}$ & 0.72 & 0.76 \\
\hline CF6. I think if someone gets colorectal cancer, he/she will die soon. & 0.70 & 0.75 \\
\hline CF15. I think colorectal cancer will kill me no matter when it is found and how it is treated. & 0.67 & 0.75 \\
\hline CF7. I think if someone gets colorectal cancer, that's the way he/she was meant to die. & 0.70 & 0.74 \\
\hline CF5. I think if someone gets colorectal cancer, it was meant to be. & 0.74 & 0.73 \\
\hline CF2. I think if someone has colorectal cancer, it is already too late to receive treatment. & 0.68 & 0.72 \\
\hline $\begin{array}{l}\text { CF4. I think if someone is meant to get colorectal cancer, he/she will get it regardless of } \\
\text { what he/she does. }\end{array}$ & 0.70 & 0.72 \\
\hline $\begin{array}{l}\text { CF14. I think if someone is meant to have colorectal cancer, he/she will get bowel cancer } \\
\text { regardless of whether he/she eats healthy foods. }\end{array}$ & 0.66 & 0.65 \\
\hline $\begin{array}{l}\text { CF10. I think some people do not want to know if they have colorectal cancer because } \\
\text { they do not want to know they may be dying from it. }\end{array}$ & 0.60 & 0.61 \\
\hline $\begin{array}{l}\text { CF3. I think someone can eat fatty foods all of their life, and if he/she is not meant to get } \\
\text { colorectal cancer, he/she won't get it. }\end{array}$ & 0.62 & 0.60 \\
\hline CF18. I think there is little one can do to prevent cancer. & 0.56 & 0.54 \\
\hline $\begin{array}{l}\text { CF1. I think if someone is meant to have colorectal cancer, he/she will get colorectal cancer } \\
\text { despite the type of food he/she eats. }\end{array}$ & 0.55 & 0.50 \\
\hline CF16. I think it is fate to get cancer. & 0.56 & 0.50 \\
\hline $\begin{array}{l}\text { CF8. I think getting checked for colorectal cancer makes people scared that they may really } \\
\text { have colorectal cancer. }\end{array}$ & 0.45 & 0.44 \\
\hline HF11. I often feel helpless in dealing with the problems of life. & 0.50 & 0.42 \\
\hline CF17. I think cancer is always fatal. & 0.34 & 0.28 \\
\hline HF12. There is really no way I can solve some of the problems I have. & 0.41 & 0.29 \\
\hline \multicolumn{3}{|l|}{ Factor 2 (health fatalism) } \\
\hline HF9. How long I live is a matter of luck. & 0.74 & 0.92 \\
\hline HF8. I think destiny or fate is determined by God. & 0.74 & 0.82 \\
\hline HF7. I think health or illness is determined by God. & 0.72 & 0.75 \\
\hline HF6. I will die when I am fated to die. & 0.71 & 0.73 \\
\hline HF10. I will stay healthy if I am lucky. & 0.62 & 0.70 \\
\hline HF5. How long I live is predetermined. & 0.75 & 0.67 \\
\hline HF4. I think health or illness is a matter of fate. & 0.70 & 0.62 \\
\hline EC10. Luck has a lot to do with whether I am healthy or ill. & 0.53 & 0.58 \\
\hline HF3. Life is predetermined. & 0.66 & 0.55 \\
\hline EC9. It is solely up to God to decide if I am healthy or ill. & 0.46 & 0.43 \\
\hline HF2. What will happen will happen regardless of what I do. & 0.54 & 0.40 \\
\hline HF1. I cannot control life and death. & 0.41 & 0.27 \\
\hline \multicolumn{3}{|l|}{ Factor 3 (crisis health orientation and external control) } \\
\hline EC6. There is nothing that I can do to detect health problems early. & 0.68 & 0.88 \\
\hline EC5. I have little influence over the things that happen to me. & 0.62 & 0.80 \\
\hline EC7. There is nothing that I can do to detect colorectal cancer early. & 0.59 & 0.79 \\
\hline
\end{tabular}


Table 4 Confirmatory factor analysis of the cultural belief scales (Continued)

\begin{tabular}{lll}
\hline Scale and items & Corrected item-Total correlation & Factor loadings \\
\hline EC4. Other powerful people decide when I should be screened for health problems. & 0.61 & 0.62 \\
EC2. Friends decide when I should be screened for health problems. & 0.63 & 0.47 \\
EC8. Identifying health problems early is a matter of chance. & 0.44 & 0.50 \\
EC3. Health care providers, such as the doctor, decide when I should be screened & 0.50 \\
for health problems. & 0.45 & 0.42 \\
CHO2. Planning for regular health screenings is not important. & & 0.36 \\
CHO3. As long as I am feeling well now, it is not important for me to have regular & 0.37 \\
health screenings. & 0.25 \\
EC1. My family members decide when I should be screened for health problems. & \\
CHO1. I only need to see my health care provider when I am sick. & 0.69 \\
Factor 4 (preventive health orientation and internal control) & 0.69 \\
PHO1. Being healthy is important for my future. & 0.68 \\
PHO2. It makes sense to take care of my health now so I can be healthy in the future. & 0.21 \\
PHO3. It is important for me to do things now to prevent health problems. & 0.62 \\
IC1. I can make a difference in my health by detecting problems early. & 0.55 \\
PHO4. Identifying health problems early is important to me. & 0.31 \\
PHO5. It is important for me to plan to have CRC screening. & 0.48 \\
IC2. I should take it upon myself to find health problems early. & 0.52 \\
IC3. Finding health problems early is my responsibility. & 0.47 \\
IC4. I have a lot to do with finding health problems early. & 0.92 \\
\hline
\end{tabular}

CF cancer fatalism, $H F$ health fatalism, EC external control, $C H O$ crisis health orientation, $P H O$ preventive health orientation, IC internal control

reliable and valid for Koreans through the rigorous instrument adaptation and validation processes.

As shown in Table 2, EFA revealed that all items for cancer fatalism, including three cancer fatalism items that were adapted from the health fatalism scale (CF 16, 17, and 18), loaded on the cancer fatalism construct, but two health fatalism items (HF11 and 12) loaded on the cancer fatalism construct and two external control items (EC 9 and 10) loaded on the health fatalism construct. A feature of the cancer fatalism construct was helplessness or powerlessness due to negative health consequences [15]; thus, HF11 and 12 items related to helplessness could be indicators of cancer fatalism. Additionally, another feature of health fatalism was lack of control over external events [12]; thus, the EC9 and 10 items related to external control by God or luck could be indicators of health fatalism.
Although low total explained variance using a 4-factor model $(48.12 \%)$ was reported in this study, methodological studies investigating belief scales about cancer screening have reported similar explained variance. For example, Lee et al. [15] examined the cultural belief scales among Korean Americans and conducted an exploratory factor analysis using all of the cultural belief items, resulting in 5 factors accounting for $46.55 \%$ of the variance. The five-factor model (cancer fatalism, health fatalism, health temporal orientation and internal control, external control, physical space) explained 7.28, 6.05, 4.89, 4.31, and $3.40 \%$ of the variance, respectively [15]. Rawl [33] also reported that explained variance for the 2-factor model factor analyses (benefits and barriers scales of fecal occult blood test, flexible sigmoidoscopy, colonoscopy) was 34, 33 , and 33\%, respectively. Champion [34] reported a 6-factor model (confidence, barriers, susceptibility, health

Table $\mathbf{5}$ Internal consistency reliability of the cultural belief subscales

\begin{tabular}{lllll}
\hline Scale & Number of items & Mean of items & Mean of item SDs & Cronbach's alpha \\
\hline Factor 1. Cancer fatalism & 20 & 2.36 & 0.78 & 0.93 \\
Factor 2. Health fatalism & 12 & 2.50 & 1.05 & 0.90 \\
Factor 3. Crisis health orientation and external control & 11 & 2.23 & 0.86 & 0.82 \\
Factor 4. Preventive health orientation and internal control & 9 & 3.95 & 0.70 & 0.83 \\
\hline
\end{tabular}


motivation, seriousness, and barriers) for breast cancer screening behaviors, finding a total of $45.7 \%$ (16.7, 9.5, 8.0, $6.7,6.2$, and $4.6 \%$, respectively). The low total variance of the model in this study may have resulted from cultural belief scales having many items (a total of 52 items) measuring multivariate variables. Items with low factor loadings were included in the model because the purpose of this study was to adapt and validate existing cultural belief scales for CRC screening among Koreans in the Republic of Korea.

Surprisingly, factor 3 consisted of items associated with crisis health orientation and external control, and factor 4 consisted of items associated with preventive health orientation and internal control, which may show that health temporal orientation and personal control were not separate but were intertwined in Koreans' cognition and mind. Regarding factor 3, people who had crisis health orientation believed that symptoms are cues to seek health care. Thus, they did not go to medical facilities for cancer screening without having symptoms, but they would undergo cancer screening if external people, such as health care providers, encouraged them to undergo cancer screening. Similarly, regarding factor 4, people with a preventive health orientation believed that they should undergo cancer screening to detect health problems early. This result is similar to that in Lee et al.'s study [15], which tested cultural belief scales in Korean Americans, and health temporal orientation and internal control were loaded as one factor. Additionally, the CFA revealed that items with high factor loadings > 0.4 were related to the external control on factor 3 (Table 4). Similarly, most of the dominant factor 4 items with factor loadings $>0.4$ were associated with the preventive health orientation.

The present study revealed significant implications through several methodological steps. First, the items in the EFA and CFA with factor loadings $<0.4$ in the present study should be considered for removal from the scale. Findings from individual interviews were helpful for understanding relationships between poor-performing items and constructs. Regarding cancer fatalism, CF 17 "I think cancer is always fatal" performed poorly in both the EFA and CFA. Based on the interviews, Korean participants believed that cancer was not always fatal because cancer could be treatable when it was found early, which caused inconsistency between the item and construct. Furthermore, interestingly, both EFA and CFA showed that the item $\mathrm{CHO} 1$, "I only need to see my health care provider when I am sick", was poorly performed with respect to factor 3, which was in accordance with our previous study in Korean Americans [15]. CHO 1 may be unclear regarding whether to measure Koreans' awareness or actual behavior. All of the participants in the individual interviews agreed that they should see health care providers when they did not have symptoms, but they did not actually see health care providers until they were sick. There were differences between Koreans' awareness and actual behaviors regarding health care utilization. In this case, item contents should measure both awareness and actual behaviors in future practice and research. Second, traditional cultural beliefs, including crisis or preventive health orientation toward health care use, and internal or external control, were investigated in the present study. Theoretically, these beliefs are separate; however, in reality, these beliefs are intertwined in Koreans. Further research needs to examine traditional Korean cultural beliefs to increase the understanding of the relationships among these beliefs and cancer screening behaviors. Also, future research needs to refine instruments, retest reliability and validity, and finalize belief scales.

While the present study provided important findings regarding the cultural belief scales in Koreans, we advise caution in interpretation of the findings due to research limitations. First, limitations regarding generalizability of the study results should be acknowledged due to the use of a convenience sample of Koreans who resided in a metropolitan city in the Republic of Korea. Second, the present study examined only EFA and CFA; thus, other validation methods for cultural belief scales need to be investigated to examine them more accurately.

\section{Conclusion}

This is the first study that adapted and validated theory-based cultural belief scales regarding CRC screening among Koreans. The results of the present study showed that four-factor cultural belief scales were culturally sensitive, reliable and valid for Koreans. The final cultural belief scales could be used to identify cultural beliefs more accurately and specifically, as well as to develop effective interventions to increase CRC screening behaviors among Koreans in the Republic of Korea.

\section{Additional file}

Additional file 1: English version of the cultural belief scales about colorectal cancer screening. (DOCX $31 \mathrm{~kb}$ )

\footnotetext{
Abbreviations

CF: Cancer fatalism; CFA: Confirmatory factor analysis; CFI: Comparative fit index; CHO: Crisis health orientation; Cl: Confidence interval; CMIN/DF: Chisquare minimum/degree of freedom; CRC: Colorectal cancer; EC: External control; EFA: Exploratory factor analysis; GFI: Goodness of fit index; HF: Health fatalism; IC: Internal control; NFI: Normed fit index; PHO: Preventive health orientation; RMSEA: Root mean square error of approximation;

SEM: Structural equation modeling; SRMR: Standardized root mean square residual; US: United States
}

Acknowledgements

Not applicable. 


\section{Funding}

This research was supported by Basic Science Research Program through the National Research Foundation of Korea (NRF) funded by the Ministry of Education (NRF-2015R1D1A1A01058169). The funding for this study supported data collection, analysis, interpretation, and writing manuscripts.

\section{Availability of data and materials}

The datasets analyzed during the current study are available from the corresponding author on reasonable request.

\section{Authors' contributions}

SL has made contributions to design, data analysis using statistical methods, data interpretation and drafted this manuscript. The sole author, SL, read and approved the final manuscript.

\section{Ethics approval and consent to participate}

The protocols of the present study were approved by the institutional review board of the Chosun University. Data were collected anonymously. Informed consent in written or verbal form about the purpose and procedures of the study, estimated risks and benefits, privacy protection and confidentiality, as well as their right to freely withdraw from the study at any time was obtained from all participants. Verbal consent was obtained from some participants with confidentiality issues (e.g., some did not want to reveal their names with signatures in written form) after the principal investigator or research assistants explained the consent statement in the present of a witness, such as the researcher or other study participants.

\section{Consent for publication}

Not applicable.

\section{Competing interests}

The author declares that she has no competing interests.

\section{Publisher's Note}

Springer Nature remains neutral with regard to jurisdictional claims in published maps and institutional affiliations.

Received: 6 February 2018 Accepted: 20 November 2018

\section{Published online: 29 November 2018}

\section{References}

1. National Cancer Information Center. Cancer incidence rates. 2017. https:// www.cancer.go.kr/lay1/S1T639C641/contents.do. Accessed 2 Feb 2018

2. National Cancer Information Center. Cancer incidence rates by ages. 2017. http://www.cancer.go.kr/mbs/cancer/subview.jsp?id=cancer_040103000000. Accessed 2 Feb 2018.

3. National Cancer Information Center. Colorectal cancer screening recommendations. Korea: National Cancer Center; 2017.

4. Ministry of Health and Welfare. The National Cancer Screening Rates. 2017. http://www.index.go.kr/potal/main/EachDtPageDetail.do?idx_cd=1440 Accessed 2 Feb 2018.

5. Kang Y, Son H. Gender differences in factors associated with colorectal cancer screening: a national cross-sectional study in Korea. Asia Pac J Public Health. 2017:29(6):495-505.

6. Myong J, Kim H. Impacts of household income and economic recession on participation in colorectal cancer screening in Korea. Asian Pac J Cancer Prev. 2012;13:1857-62.

7. Bae N, Park S, Lim S. Factors associated with adherence to fecal occult blood testing for colorectal cancer screening among adults in the Republic of Korea. Eur J Oncol Nurs. 2014;18:72-7.

8. Kandula NR, Wen M, Jacobs EA, Lauderdale DS. Low rates of colorectal, cervical, and breast cancer screening in Asian Americans compared with non-Hispanic whites: cultural influences or access to care? Cancer. 2006; 107(1):184-92.

9. Lee YY, Jun JK, Suh M, Park B, Kim Y, Choi KS. Barriers to cancer screening among medical aid program recipients in the Republic of Korea: a qualitative study. Asian Pac J Cancer Prev. 2014;15(2):589-94.

10. Kang HS, Thomas E, Kwon BE, Hyun M, Jun EM. Stages of change: Korean women's attitudes and barriers toward mammography screening. Health Care Women Int. 2008;29:151-64.
11. Suh M, Choi KS, Lee YY, Park B, Jun JK. Cancer screening in Korea, 2012: results from the Korean National Cancer Screening Survey. Asian Pac J Cancer Prev. 2013;14(11):6459-63.

12. Lee S-Y, Lee E. Korean Americans' beliefs about colorectal cancer screening Asian Nurs Res. 2013;7(2):45-52.

13. Suh EE. The socio-cultural context of breast cancer screening among Korean immigrant women. Cancer Nurs. 2008;31(4):E1-10.

14. Son H, Kam S, Park K, Kim J, Kim R, Park S. The factors associated with changes in the stage of breast cancer screening behavior among the woman who are eligible for the Korean National Cancer Screening Program. J Prev Med Public Health. 2009:42(2):109-16.

15. Lee S-Y, Lee EE, Aranda F. Instrument adaptation, modification, and validation for cultural beliefs about colorectal cancer screening among Korean Americans. Cancer Nurs. 2018. https://doi.org/10.1097/NCC. 0000000000000523

16. Russell KM, Perkins SM, Zollinger TW, Champion V. Sociocultural context of mammography screening use. Oncol Nurs Forum. 2006:33(1):105-12.

17. Giger JN, Davidhizer RE. Transcultural nursing: assessment and intervention. St. Louis, MO: Mosby; 1999

18. Powe BD. Fatalism among elderly African Americans: effects on colorectal cancer screening. Cancer Nurs. 1995;18(5):385-92.

19. Polit DF, Beck CT. The content validity index: are you sure you know what's being reported? Critique and recommendations. Res Nurs Health 2006; 29(5):489-97.

20. Hatcher L. A step-by-step approach to using the SAS system for factor analysis and structural equation modeling. SAS Institute, Inc: Cary, NC; 1994

21. Hutcheson G, Sofroniou N. The multivariate social scientist: introductory statistics using generalized linear models. Thousand Oaks, CA: Sage Publications; 1999

22. Comrey AL, Lee HB. A first course in factor analysis. Eribaum: Hilsdale, NJ; 1992.

23. Kline RB. Principles and practice of structural equation modeling, 2nd edn New York: The Guilford Press; 2005.

24. IBM Corp. IBM SPSS Statistics for Windows, Version 24: Armonk, NY, IBM Corp.; 2016.

25. Arbuckle JL. Amos version 23.0. In. Chicago: IBM SPSS; 2014.

26. Tabachnick BG, Fidell LS. Using multivariate statistics. 4th ed. Boston: Allyn and Bacon; 2001.

27. Capik C, Gozum S. Development and validation of health beliefs model scale for prostate cancer screenings: evidence from exploratory and confirmatory factor analyses. Eur J Oncol Nurs. 2011;15:478-85.

28. Nunnally JC. Psychometric theory. 2nd ed. New York: McGraw-Hill; 1978.

29. DeVellis RF. Scale development: theory and applications. 4th ed. Newbury Park, CA: Sage; 2016.

30. Norton R. Measuring marital quality: a critical look at the dependent variable. J Marriage Fam. 1983:45(1):141-51.

31. Wood ND, Gnonhosou DC, Bowling J. Combining parallel and exploratory factor analysis in identifying relationship scales in secondary data. Marriage Fam Review. 2015;51(5):385-95.

32. Schumaker RE, Lomax RG. A beginner's quide to strcutural equation modeling. 2nd ed. Malwah, NJ: Lawrence Erlbaum Associates; 2004

33. Rawl S, Champion V, Menon U, Loehrer PJ Sr, Vance GH, Skinner CS. Validation of scales to measure benefits of and barriers to colorectal cancer screening. J Psychosoc Oncol. 2001;19(3):47-63.

34. Champion V. Instrument refinement for breast cancer screening behaviors. Nurs Res. 1993;42(3):139-43.

Ready to submit your research? Choose BMC and benefit from:

- fast, convenient online submission

- thorough peer review by experienced researchers in your field

- rapid publication on acceptance

- support for research data, including large and complex data types

- gold Open Access which fosters wider collaboration and increased citations

- maximum visibility for your research: over $100 \mathrm{M}$ website views per year

At $\mathrm{BMC}$, research is always in progress.

Learn more biomedcentral.com/submission 